\title{
Analysis on GPR Echo Signal Enhancement Algorithm based on Subspace Sparsity Deconvolution
}

\author{
Yang Yong $^{12, *}$, Du Yanliang ${ }^{2}$ Zhao Weigang ${ }^{2}$, Wang Baoxian ${ }^{2}$ and Zhao Hao ${ }^{3}$ \\ ${ }^{1}$ School of Mechanical, Electronic and Control Engineering, Beijing Jiaotong University, Beijing 100044, China \\ ${ }^{2}$ The Key Laboratory for Health Monitoring and Control of Large Structures in Hebei Province, Shijiazhuang Tiedao University, \\ Shijiazhuang 050043, China \\ ${ }^{3}$ Department of Urban \& Civil Engineering, Ibaraki University, Hitachi 316-8511, Japan
}

Received 29 May 2016; Accepted 22 November 2016

\begin{abstract}
Strong reflection interference of rebar is the main barrier against ground-penetrating radar (GPR) detection of internal diseases in reinforced concrete structure. Deconvolution can strengthen GPR echo data through acquiring structural layer data. However, existing deconvolution echo signal processing algorithms cannot be applied to process the GPR echo data because of the hypothesis that the wavelet is in the minimum phase system, which is inconsistent with the attenuation of GPR wavelet during two-way travel time. To resolve this problem, a new subspace sparsity deconvolution algorithm was proposed in this paper to enhance the echo signal of rebar lower diseases. A wavelet signal attenuation model of layered structure was firstly established to analyse the influencing factors of disease echo signal attenuation under different conditions. On this basis, the sparsity deconvolution algorithm based on wavelet iteration was placed to enhance GPR echo signal quickly by using the constraint condition of subspace errors. The processing of GPR forward simulation data also reveals that the proposed algorithm can inhibit noises effectively and acquire layer information from the concrete structure when the local signal-to-noise ratio is larger than $-19.7 \mathrm{~dB}$. The proposed algorithm is clearly superior to the existing automatic gain control algorithm with respect to the echo enhancement effect of GPR. Results show that this method overcomes the minimum phase restriction of deconvolution and it is of great significance to detect and recognize inter-layer diseases of high-speed railway ballastless tracks.
\end{abstract}

Keywords: Deconvolution, Subspace, Ground penetrating radar, Signal enhancement, Ballastless track disease detection

\section{Introduction}

The approximate temperature expansion curve and the high binding strength between rebar and concrete increase the tensile and compressive strengths of concrete, making the reinforced concrete (RC) structure widely applicable in infrastructure construction. Taking the CRTS-II type ballastless track as an example, which consists of track plate, cement asphalt (CA) mortar and support layer, its health status directly affects the running safety of high-speed train. However, considering the long-term effect of load, different degrees and types of diseases will develop in $\mathrm{RC}$ structure, thus affecting its normal operation. Meanwhile, the concealing characteristic of diseases in the $\mathrm{RC}$ structure increases the detection difficulty. Therefore, a way to detect internal diseases of RC structure quickly and accurately is the key to infrastructure monitoring.

Ground-penetrating radar (GPR) [1-2] is an important method to detect the internal diseases of $\mathrm{RC}$ structure for its fast, continuous and non-destructive characteristics. In theory, GPR detects underground target by taking advantage of the electromagnetic wave reflecting characteristic on surfaces of different dielectric parameters. As a good conductor, rebar is of strong reflection for electromagnetic wave, the reflectivity is $100 \%$, the transmittance is $0 \%$, and

- E-mail address: sherry_0768@163.com

ISSN: $1791-2377$ @ 2016 Eastern Macedonia and Thrace Institute of Technology. All rights reserved. only a small part of energy continues to propagate downward by way of diffraction. This characteristic means that disease echo signal with rebar coverage is smaller than that without rebar coverage, which makes disease recognition more difficult. Therefore, enhancing the disease echo signal and eliminating the influences of rebar coverage is a big challenge to detect diseases of RC structure by GPR.

On this basis, a subspace sparsity deconvolution algorithm is proposed to analyze and discuss the characteristics of disease echo signal covered by rebar. The proposed algorithm can enhance disease echo signal and eliminate the influences of strong reflection interference.

\section{State of the art}

Many existing commercial software (e.g., GSSI Radon and IDS GRE) enhance the GPR signal mainly through automatic gain control (AGC) methods, such as Standard AGC and Gaussian-tapered AGC [3-5]. The Standard AGC amplifies the signal in the time window proportionally according to the numerical relationship of echo intensity between signal in the time window and signal in the whole sampling interval. Gaussian-tapered AGC adjusts the center data of the Gaussian conical sliding window and achieves the AGC of GPR echo data according to changes of the rootmean-square amplitude. These algorithms amplify target echo and noise signal by using the same proportionality 
coefficient, thus failing to increase the signal-to-noise ratio (SNR).

Another algorithm assumes that the underground structure is a sparsity-layered structure, and the echo signal is the convolution of layered coefficient and launched wavelet. It gets the sparsity expression of the layered structure by calculating the deconvolution factor and enhances the interlayer reflection echo signal by executing a normalization process. Such algorithm is widely used in seismic signal processing because it increases the probe resolution and improves profile records. Common deconvolution algorithms include frequency-domain deconvolution [6], spiking deconvolution [7-8], predictive deconvolution [9-11], minimum entropy deconvolution (MED) [12-14], and homomorphic deconvolution [15]. Frequency-domain deconvolution acquires interlayer reflection-coefficient directly under a known wavelet in accordance to the relationship between the time-domain convolution and the frequency-domain product. Spiking deconvolution [7-8] directly acquires the deconvolution factor with respect to the least square and realizes interlayer echo positioning under the hypothesis of a known wavelet. However, the condition number of Toeplitz matrix is large owing to the dispersive property of the underground medium and sampling error, which causes a large error and a high false alarm rate in the process of calculating reflection coefficient. Predictive deconvolution [9-11] assumes that wavelet is the minimum phase system and noise is white. It gains the Toeplitz matrix by replacing wavelet autocorrelation with echo signal autocorrelation and thereby calculates the reflection coefficient. Considering that GPR echo signal is a typical non-minimum phase system, the predictive deconvolution is inapplicable to GPR signal deconvolution calculation. MED [12-14] assumes that the reflection coefficient is composed of sparse spike pulses, and the wavelet length is shorter than the minimum interval of reflection interfaces, which indicates that no echo overlapping exists in different reflection layers. However, GPR is mainly used to recognize superficial targets with small intervals and echo overlapping occurs frequently. Therefore, MED cannot apply to GPR signal deconvolution calculation. Homomorphic deconvolution [15] assumes that wavelets are relatively stable in the logarithmic statistical law of echo signal, where reflection coefficient is a random signal. The mean of random signal is close to zero given adequate samples. Under this circumstance, the statistical mean of echo signals is viewed as the wavelet signal, and thus the interlayer reflection coefficient is calculated. Moreover, all the discussed deconvolution algorithms calculate the reflection coefficient under the hypothesis that the wavelet is fixed, which disagrees with the changes of wavelet form during the transmission of GPR electromagnetic wave in loss medium.

On the basis of the traditional convolution model, Margrave et al. [16-17] proposed the Gabor convolution model for attenuation wavelet estimation in 1999. This model is built under the hypothesis that the interlayer reflection coefficient is a white noise sequence and that wavelet has a fixed attenuation coefficient. But in the process of electromagnetic wave transmission, wavelet is a time-varying signal and the attenuation coefficient is likewise relevant to transmission distance, reflection coefficient, and interlayer structure, which is contradicted to the hypothesis of fixed attenuation coefficient in Gabor convolution model. Therefore, the Gabor deconvolution algorithm cannot be applied directly to GPR layer recognition and signal enhancement.

Owing to the shortages of the existing deconvolution algorithm, the non-minimum phase sparsity deconvolution algorithm for GPR is explored. This proposed algorithm can be used to acquire accurate layer information, reconstruct GPR echo data, and enhance the disease echo signal. The rest of this paper is organized as follows. Section 3 establishes the wavelet attenuation model under rebar coverage, analyzes the influencing factors of wavelet, studies the subspace sparsity deconvolution algorithm principle and its frame structure, and then constructs a multilayer medium model and realizes forward simulation based on finite-different time-domain (FDTD). Two conditions with and without echo overlapping are processed by the subspace sparsity deconvolution algorithm, thus verifying the feasibility of the proposed algorithm. Section 4 analyses the influences of upper rebar to the lower void echo signal and discusses the robustness of the algorithm. Section 5 presents the conclusion.

\section{Methodology}

\subsection{Wavelet attenuation model}

In Fig. 1, the A-Scan GPR echo signal $(s)$ in the RC structure with diseases includes the direct wave $\left(s_{1}\right)$, strong reflection signal of rebar $\left(s_{2}\right)$, void echo signal $\left(s_{3}\right)$, and random noise $(n)$.

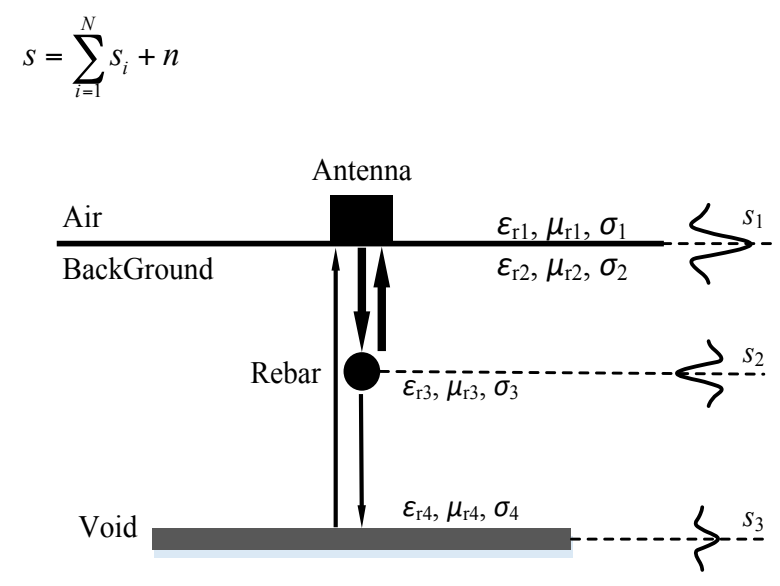

Fig. 1. GPR echo in the multilayer structure

In Fig. $1, \varepsilon_{r i}$ is the relative permittivity of the $i^{\text {th }}$ layer, $\mu_{r i}$ is the relative permeability of the $i^{t h}$ layer, $\sigma_{i}$ is the conductivity of the $i^{\text {th }}$ layer, and $N$ is the total layer number in the model.

Under ideal conditions, the electromagnetic wave emitted by GPR is a determined wavelet $b(t)$ (e.g., Ricker wavelet). The reflection coefficient $\left(r_{i}\right)$ is related to the electrical parameters and can be expressed as

$$
r_{i}=\frac{\eta_{i+1}-\eta_{i}}{\eta_{i+1}+\eta_{i}}
$$


where $\eta_{i}$ and $\eta_{i+1}$ are wave impedances of the $i^{\text {th }}$ layer and the $(i+1)^{t h}$ layer $\left(\eta_{i}=\sqrt{\mu_{i} / \varepsilon_{i}}\right.$ and $\left.\eta_{i+1}=\sqrt{\mu_{i+1} / \varepsilon_{i+1}}\right)$.

If the two-way travel time of the interface reflection echo recovered by the GPR antenna is $t_{i}$, then the reflection coefficient function $(g(t))$ of different interfaces can be expressed as the time function:

$$
g(t)=\sum_{i=1}^{N} r_{i} \delta\left(t-t_{i}\right)
$$

Owing to the differences of the background medium and structure, the influencing factors of the interlayer echo signal attenuation of electromagnetic wave from the GPR are different:

(1) For lossless medium, when echoes of different interfaces are independent and there are no rebar coverages and echo overlappings, the echo signal of GPR can be expressed as follows:

$$
s(t)=b(t) * g(t)=\int_{t=0}^{\infty} b(\tau) g(t-\tau) d \tau
$$

(2) For loss medium, when echoes of different interfaces are independent and there are no rebar coverages and echo overlappings, the amplitude attenuation and the two-way travel time $(t)$ have an exponential relationship. The echo signal of GPR can be expressed as

$$
s(t)=b(t) * g(t) e^{-a t}=\int_{t=0}^{\infty} b(\tau) g(t-\tau) e^{-a(t-\tau)} d \tau
$$

where $\alpha$ is the attenuation coefficient of the echo amplitude. (3) For loss medium, when echoes of different interfaces are independent and there is a rebar coverage with reflection coefficient $r=-1$ as shown in Fig. 1. Two extreme conditions are considered. When the rebar diameter is infinite, no diffracted wave exists and the void echo signal approaches zero. With the reduction of rebar diameter, the energy of the diffraction wave increases and the void echo signal increases accordingly. Therefore, it is hypothesized that the rebar diameter is inversely proportional to the amplitude of the void echo signal. The inversely proportional ratio is the diffraction coefficient (DC), which is expressed by $\beta(t)$. Under these conditions, the echo signal of GPR can be rewritten as

$$
s(t)=b(t) * \beta(t) g(t) e^{-a t}=\int_{t=0}^{\infty} b(\tau) \beta(t-\tau) g(t-\tau) e^{-a(t-\tau)} d \tau
$$

The $\beta(t)$ is the function about time and satisfies $0 \leq \beta(t) \leq 1$. Given a rebar diameter, $\beta(t)$ is a constant. When the rebar diameter is infinite, it is expressed as $\beta(t)=0$. When the rebar diameter is zero, the electromagnetic wave of void reflection interface is a direct wave and $\beta(t)=1$. Eq. (6) degrades into Eq. (5), where $\beta(t)=1$, and Eq. (6) degrades into Eq. (4) where $\beta(t)=1$ and $\alpha=0$. Therefore, Eqs. (4) and (5) are viewed as the special form of Eq. (6).
(4) For loss medium, echoes of different interfaces are independent, reflection coefficient $r \neq-1$, and some electromagnetic wave can transmit into the next layer. Its expression is the same as that of Eq. (6), except that DC is converted into the transmission coefficient.

\subsection{Deconvolution}

Deconvolution calculates the deconvolution factor that corresponds to the wavelet, thus getting signal processing method for layer information. With Eq. (4) as the example, the frequency-domain deconvolution often makes Fourier transforms to the two sides of the equation. According to the time-frequency relationship of convolution operation, Eq. (4) can be expressed as follows in frequency domain:

$$
S(\omega)=B(\omega) G(\omega)
$$

where $S(\omega), B(\omega), G(\omega)$ are the Fourier transform of $s(t), b(t), g(t)$. If the echo $s(t)$ and wavelet $b(t)$ are known, the reflection coefficient $g(t)$ can be expressed as

$$
g(t)=\operatorname{IFT}\{S(\omega) / G(\omega)\}
$$

where IFT $\{$.$\} is the inverse Fourier transform operator.$

Time-domain deconvolution algorithm often uses the relationship between convolution and related operations. For instance, the spiking deconvolution hypothesizes that wavelet is the minimum phase and the noise signal is a white noise signal. To calculate and organize the convolution $(b(-t))$ on the two sides of Eq. (4):

$$
R_{b b}(t) * g(t)=R_{b s}(t)
$$

where $R_{b b}(t)$ expresses the autocorrelation function of $b(t)$ and is a Toeplitz matrix. $R_{b s}(t)$ is the correlation matrix between $s(t)$ and $b(t)$. Thus, $g(t)$ can be expressed as

$$
g(t)=R_{b b}^{-1}(t) R_{b s}(t)
$$

where $R_{b b}^{-1}(t)$ is the inverse of matrix $R_{b b}(t)$.

Wavelet in the frequency-domain deconvolution is an estimation value that has some error with real value. Accordingly, the $g(t)$ calculated from Eq. (8) has significant errors, which means the result is not composed of monopulse but has numerous shock signals.

On the basis of the analysis in Section 3.1, the disease echo signal in RC structure has wavelet signal attenuation, which is attributed to two-way travel time of the electromagnetic wave, rebar size and coverage. This outcome contrasts with the hypothesis on the minimum phase system of the time-domain deconvolution algorithm.

\subsection{Subspace sparsity deconvolution}

An analysis of the time-domain deconvolution algorithm and frequency-domain deconvolution algorithm reveals that the existing deconvolution algorithms are inapplicable to the GPR layer information detection. Therefore, it is necessary to study the deconvolution algorithm applicable to GPR under layer sparsity conditions.

According to Eq. (6), the acquired interlayer reflection coefficient can be converted into the optimal solution of $\alpha, \beta, r$, and $N$. Considering that $\alpha, \beta, r$, and $N$ are 
coupling mutually, the optimization problem is changed into a high-dimension data problem, which is against solving. To optimize the solution and achieve fast convergence, the echo signal of the $i^{\text {th }}$ layer in the layered structure is denoted as

$$
f_{i}(t)=b(t) * \beta(t) r_{i} e^{-\alpha t} \delta\left(t-t_{i}\right)
$$

The echo signal of the $(i+1)^{t h}$ layer is

$$
f_{i+1}(t)=b(t) * \beta(t) r_{i+1} e^{-\alpha t} \delta\left(t-t_{i+1}\right)
$$

The Fourier transform of Eqs. (11) and (12) are calculated as

$$
\begin{aligned}
& F_{i}(\omega)=B(\omega) \beta\left(t_{i}\right) r_{i} e^{-\alpha t_{i}} e^{-j \omega t_{i}} \\
& F_{i+1}(\omega)=B(\omega) \beta\left(t_{i+1}\right) r_{i+1} e^{-\alpha t_{i+1}} e^{-j \omega t_{i+1}}
\end{aligned}
$$

A comparison of Eqs. (13) and (14) indicates that the amplitude ratio $(k)$ and the phase difference $(\Delta \theta)$ are

$$
k=\frac{\left|F_{i+1}(\omega)\right|}{\left|F_{i}(\omega)\right|}=\frac{\beta\left(t_{i+1}\right) r_{i+1} e^{-\alpha t_{i+1}}}{\beta\left(t_{i}\right) r_{i} e^{-\alpha t_{i}}}
$$

$$
\Delta \theta=\omega\left(t_{i+1}-t_{i}\right)
$$

The sampling time window in high-frequency GPR is often in nanoseconds, $\left(t_{i+1}-t_{i}\right) \rightarrow 0$. Therefore, the phase difference is viewed as $\Delta \theta \rightarrow 0$, and the waveforms between two layers have only one coefficient difference $k$. As a result, the nearest layer of the echo signal is used to replace the wavelet signal when calculating the next layer information. The optimization problem of $\alpha, \beta, r$, and $N$ changes to the optimal solutions of variables $k$ and $N$.

Meanwhile, the error shall be restricted when calculating the optimal solutions of $k$ and $N$ because $N$ is an unknown. When the constraint error is relatively large, the interface with small reflection coefficient will be missed and the missing alarm will be sent. However, when the constraint error is small, new reflection interfaces will be formed and a false alarm will be sent.

To overcome the above shortages, combined with the short time Fourier transform, a subspace window function is introduced.

If the window function is $w(t)$, the subspace can be expressed as follows:

$$
x(t)=s(t) w(t-\tau)
$$

Meanwhile, the support interval of the selection window function $(w(t))$ is $0<t<2 t_{0}$, if the support interval of wavelet $b(t)$ is $0<t<t_{0}$, where $0<t<t_{0}$ is the previous layer of electromagnetic wave whose time-domain coordinate of the echo peak is $t_{0} / 2$. The possible next layer of electromagnetic echo peak may occur in the interval of $\left(t_{0} / 2,3 t_{0} / 2\right)$. In this way, the problem that $N$ is an unknown in the whole interval is changed into the problem of $N(0,1)$ in the subspace $\left(t_{0} / 2,3 t_{0} / 2\right)$. When $N=0$, no new reflection layer is formed in the interval of $\left(t_{0} / 2,3 t_{0} / 2\right)$. However, when $N=1$, the new reflection layer is formed in the interval of $\left(t_{0} / 2,3 t_{0} / 2\right)$.

The electromagnetic echo overlapping between two adjacent layers (the $i^{t h}$ layer and the $(i+1)^{t h}$ layer) is shown in Fig. 2. Supposing that the wavelet after discretion is $b(n)$ and its support interval is $n=1 \ldots P$, where $P=t / \Delta t$ and $\Delta t$ is the sampling time interval. The support interval of two overlapping layers is $n=1 \ldots 2 P$. The critical echo signal overlapping that exists between the $i^{\text {th }}$ layer and the $(i+1)^{t h}$ layer is shown in Fig. 2(a). Moreover, the echo amplitude ratio between the $i^{\text {th }}$ layer and the $(i+1)^{\text {th }}$ layer is $k=0.8$. The wavelet form of the electric field component $E$ when the Ricker wavelet is used as the wavelet emitted by GPR antenna is shown in Fig. 2(b). The support interval of the wavelet has 200 sample points. The waveform overlapping between the $i^{\text {th }}$ layer and the $(i+1)^{\text {th }}$ layer under the interlayer interval of 100 sample points is shown in Fig. 2(c), where the amplitude ratio is $k=0.5$.

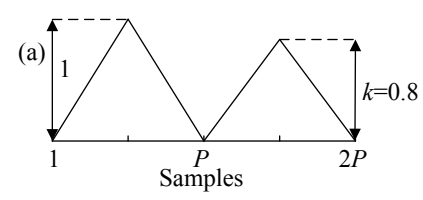

(b)

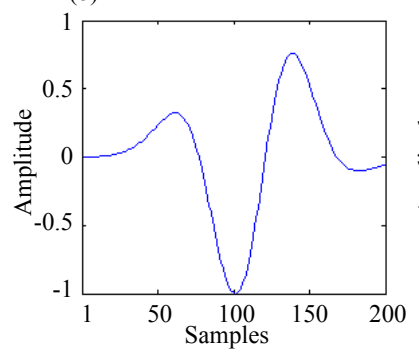

(c)

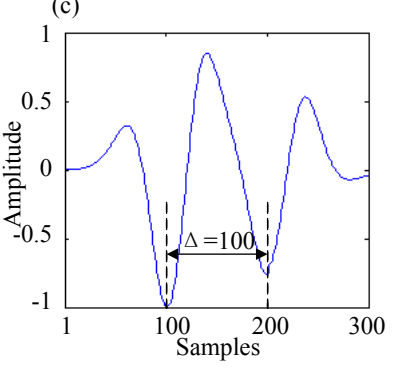

Fig. 2. Echo overlapping (a) Critical echo overlapping of two layers; (b) Ricker wavelet; (c) Overlapping under the interlayer interval of 100 sample points

\subsection{Fast algorithm implementation}

The key of fast implementing the subspace sparsity deconvolution lies in the selection of the initial wavelet, the settings of iteration step length, and constraint conditions.

1. Initial wavelet

Choosing the appropriate initial wavelet is the foundation for reducing error of layer number and acquiring accurate layer information.

Circuits in the electromagnetic wave emission antenna made by different GPR factories (e.g., GSSI, IDS and Mala) are different, which leads to different initial wavelet. In general, the electromagnetic wave emitted by GPR antenna arrives first at the air-ground interface. Considering the difference of relative permittivity between air and ground, some electromagnetic wave reflections will be received by the GPR antenna and form direct waves. As air is a kind of lossless medium, the direct wave reflects the wavelet form emitted by the GPR antenna. Therefore, the direct wave is often chosen as the initial wavelet $b(t)$.

\section{Iteration step length}

Supposing that the support interval of the current window function $w(t-\tau)$ is $\tau<t<\tau+2 t_{0}$, where $\tau$ is the start point and $\tau+2 t_{0}$ is the end point. There are two situations 
whether new reflection layers are formed on the interval of $\tau+t_{0} / 2<t<\tau+3 t_{0} / 2$.

(1) When a new reflection layer is formed, the location of the new reflection layer is $\tau+\Delta t$. The start point and end point of the new window functions are gained by updating $t=t+\Delta t$, where $0<\Delta t<t_{0} / 2$.

(2) When there is no new reflection layer, the start point and end point of the new window function are gained by updating $t=t+t_{0} / 2$.

\section{Constraint conditions}

When the sampling time window is adequately long, the GPR antenna will receive enough echoes. Each echo data can be viewed as having equal energy:

$$
\int_{t=0}^{\infty} s(t) s^{*}(t) d t=c
$$

where $c$ is a constant, $s(t)$ is the A-Scan echo signal, and * is a complex conjugate.

Eq. (18) can also be expressed as the signal emitted by GPR transmitting antenna with energy of $c$, and all energies are reflected between layers and received by the receiving antenna. Therefore, the superficial echo has a large energy, whereas a deep echo has a small energy in the GPR map. With respect to constraints, superficial echo has a big constraint value and deep echo has a small constraint value. In the RC structure, reflection echo energy of the upper layer is generally larger than the sum of the rest layers. Hence, the constraint value under initial condition is expressed as threshold $=c / 2$. Moreover, the constraint value is updated accordingly upon each update of the wavelet:

$$
\text { threshold }=\text { threshold }-\langle b(n), b *(n)\rangle / 2
$$

where $b(n)$ is the discrete form of $b(t)$. The A-Scan echo signal-processing flowchart of GPR is shown in Fig. 3.

\subsection{Forward simulation}

To verify the feasibility of the algorithm, an RC structural disease model was established for contrastive analysis of forward simulations of different algorithms under different conditions.

The test model uses the concrete structure as background, which contains rebar and void diseases at the lower part of the rebar (Fig. 4).

The model size is $(1.0 \mathrm{~m}, 0.25 \mathrm{~m})$, and the concrete medium parameters are $\left(\varepsilon_{r}, \mu_{r}, \sigma\right)=(9,1,0.1)$. The void parameters are $\left(\varepsilon_{r}, \mu_{r}, \sigma\right)=(1,1,0)$ with depth $h_{2}=0.2 \mathrm{~m}$. The rebar diameter is $d=1 \mathrm{~cm}$. The depth from rebar to the ground $\left(h_{1}\right)$ is set as a variable $\left(h_{1}=3 \mathrm{~cm}\right.$ and $\left.5 \mathrm{~cm}\right)$ to test the performances of the echo signal in subspace sparsity deconvolution algorithm under different conditions.

The forward numerical simulation is applied with the electromagnetic wave simulation software GPRMax [21-22] based on the FDTD [18-20]. The simulation parameters are listed in Table 1.

Simulation results are shown in Fig. 5. The echo signal in Fig. 5 includes direct wave, rebar echo, and void echo. The echo data when $h_{1}=5 \mathrm{~cm}$ are shown in Fig. 5(c). The extracted A-Scan echo data when rebar is above the void disease in Fig. 5(c) are shown in Fig. 5(d). The start location and peak of three interlayer echoes are clear. The echo data when $h_{1}=3 \mathrm{~cm}$ are shown in Fig. 5(a). The A-Scan echo data extracted when the rebar is above the void disease in Fig. 5(a) are shown in Fig. 5(b). The direct wave and rebar echo overlap because of the short spatial distance between them. The start position of rebar echo is ambiguous, which is against further data processing.

\begin{tabular}{|c|c|c|c|}
\hline Properties & Value & Properties & Value \\
\hline Wavelet & Ricker & $\begin{array}{c}\text { Frequency } \\
\text { /(MHz) }\end{array}$ & 2000 \\
\hline Time window/(ns) & 6 & Samples & 1007 \\
\hline Trace interval $/(\mathrm{mm})$ & 0.5 & $\begin{array}{l}\text { Grid size } \\
/(\mathrm{mm}, \mathrm{mm})\end{array}$ & $(2,4)$ \\
\hline
\end{tabular}

Table 1. Forward simulation parameters

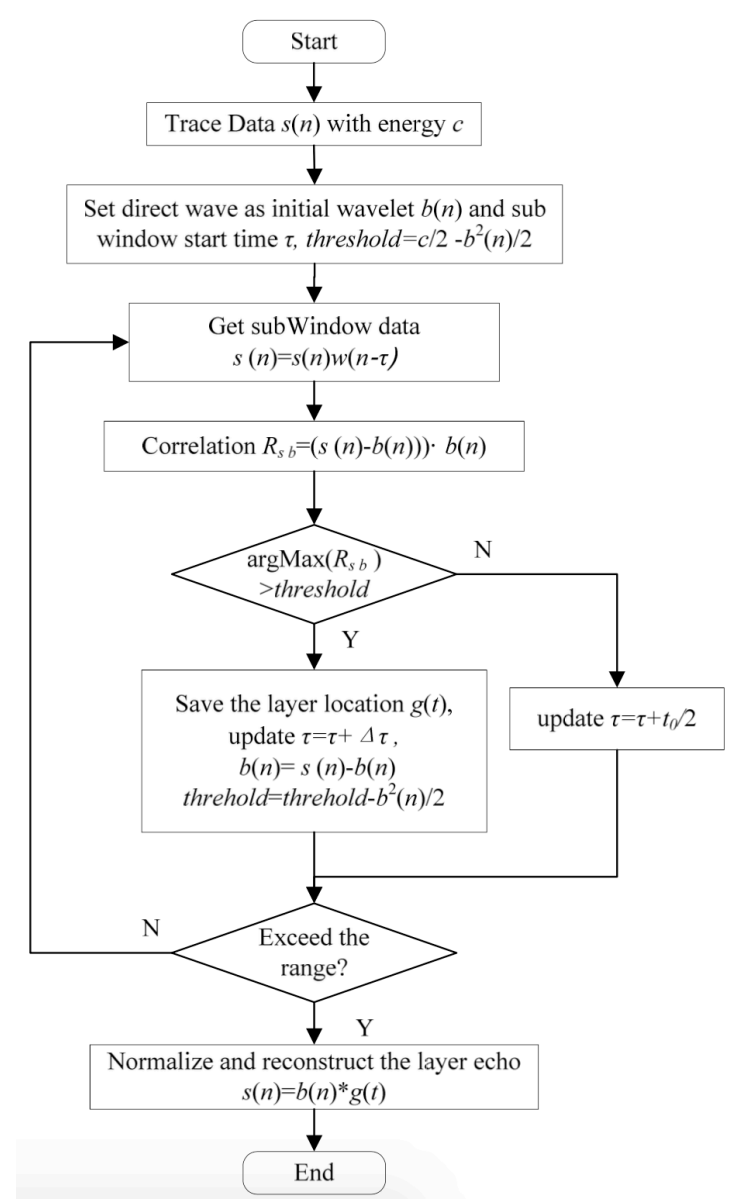

Fig. 3. Flowchart of the subspace sparsity deconvolution algorithm

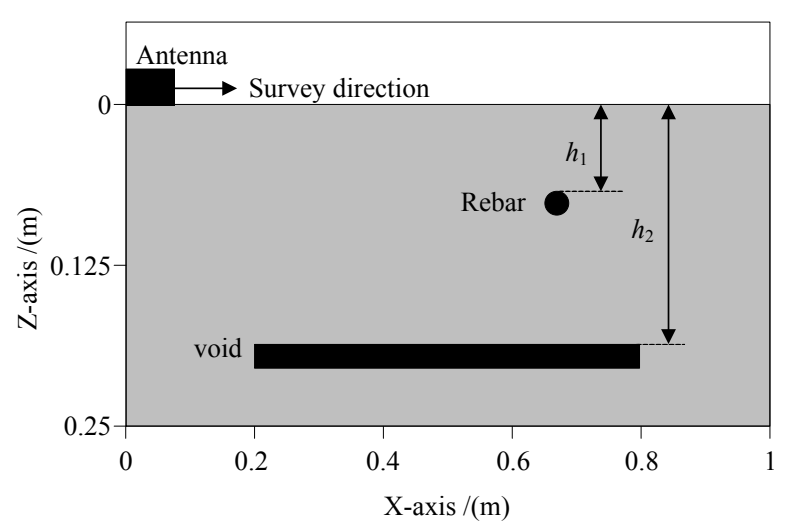

Fig. 4. Simulation test model 


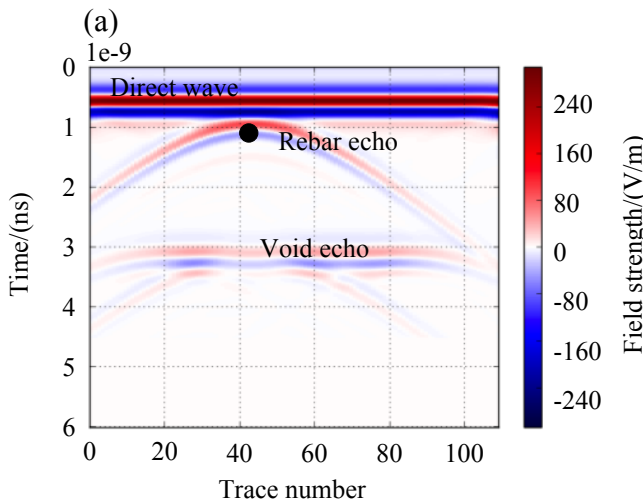

(c)

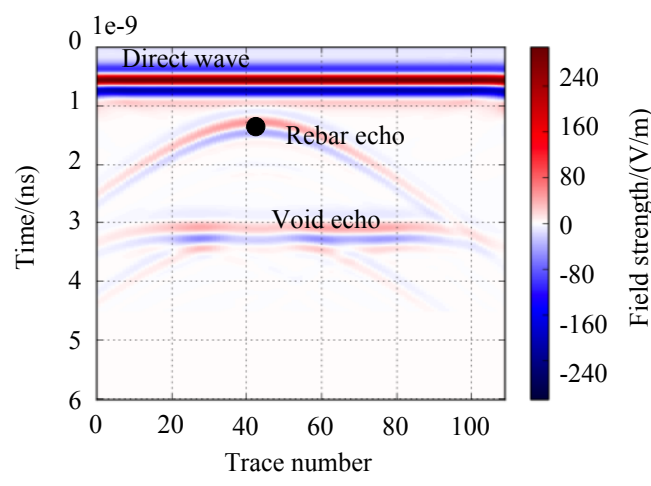

Fig. 5. Forward simulation results (a) B-Scan result with $h_{1}=3 \mathrm{~cm}$; with $h_{1}=5 \mathrm{~cm}$; (d) An A-Scan plot in (c) where trace number $=44$.

First, A-Scan echoes in Figs. 5(b) and (d) are processed by the subspace sparsity deconvolution algorithm. Results are shown in Fig. 6.
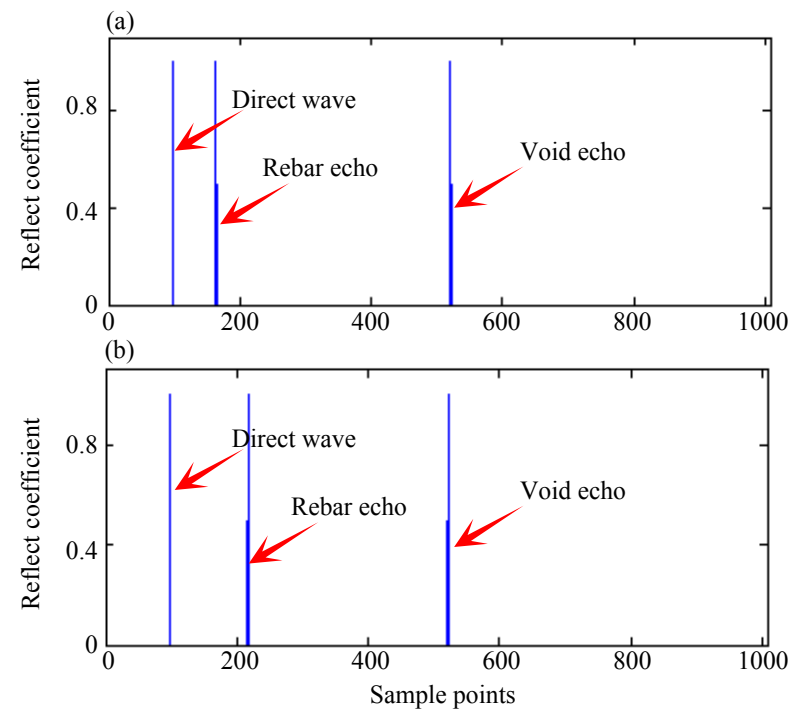

Fig. 6. Processing result with subspace sparsity deconvolution: (a) $h_{1}=3 \mathrm{~cm}$; (b) $h_{1}=5 \mathrm{~cm}$

Fig. 6 shows that the three interfaces are very clear regardless of the overlapping that exists between rebar echo and direct wave, the information about layer locations and numbers are reflected accurately. Normalization results at the overlapping of rebar echo and direct wave in Fig. 5(a) are shown in Fig.7. The direct wave, rebar echo, and void echo are explicit, and the start position of the echo has clear

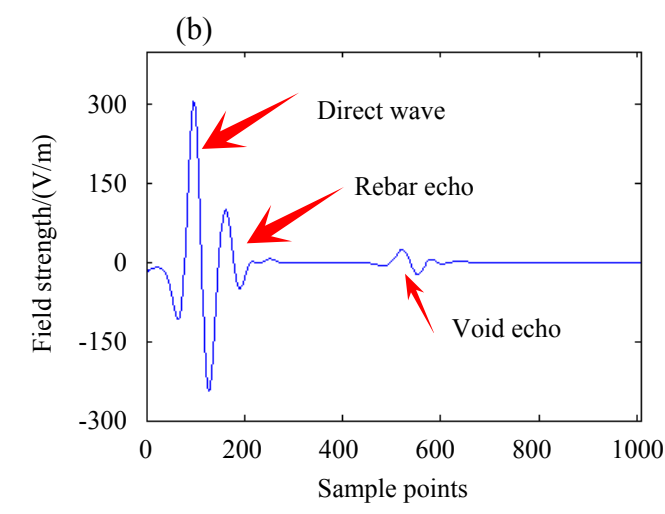

(d)

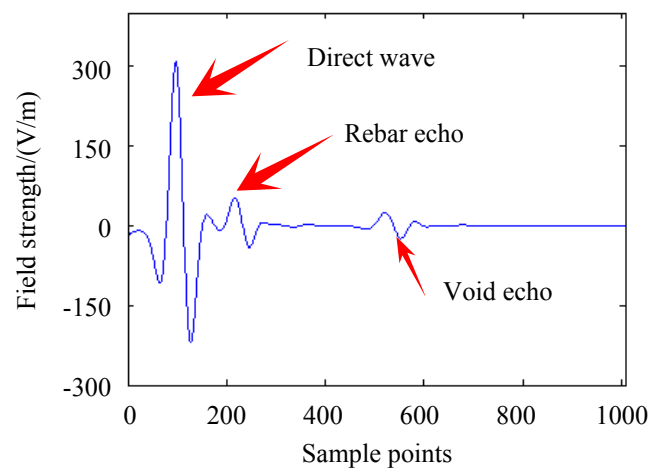

(b) An A-Scan plot in (a) where trace number $=44$; (c) B-Scan result boundaries. In particular, the void echo under rebar coverage is significantly enhanced, thus providing a good data basis for further disease recognition.

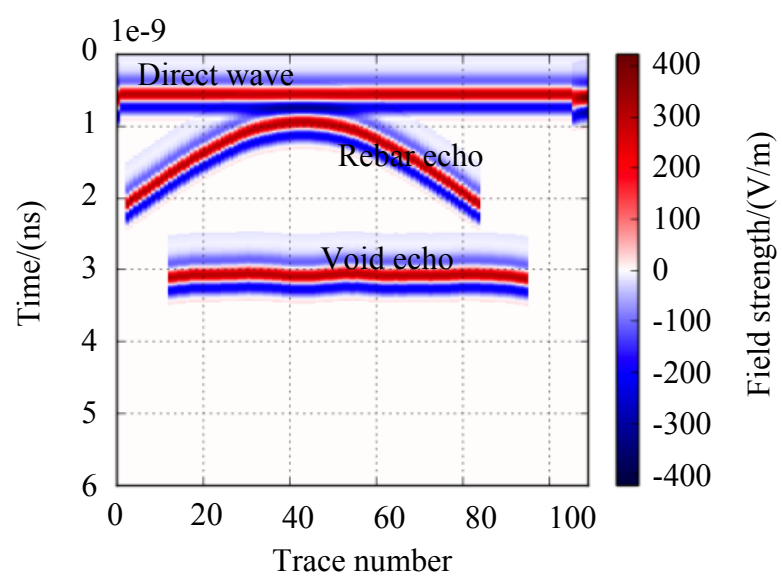

Fig. 7. Reconstructed GPR echo with $h_{1}=3 \mathrm{~cm}$

\section{Result Analysis and Discussion}

\subsection{Interference of rebar coverage to void echo}

Influences of rebar coverage on void echo are mainly manifested by echo intensity attenuation, echo delay, and waveform overlapping.

\section{Influences on void echo intensity and echo time}

Rebar is viewed as a singular point in concrete medium. The electromagnetic wave refracts and reflects on surfaces of two media. Given that the rebar is a good conductor with 
an internal electric field intensity of $E=0, \varepsilon_{r}=\infty$, then reflection coefficient $r=-1$ and refraction coefficient $\xi=0$. According to the Huygens principle, the echo on each point of the rebar can be regarded as a new wave source, and some echoes are transmitted to void disease surface through diffraction. This demonstrates that the electromagnetic wave intensity that transmits to the void surface through diffraction with rebar coverage is smaller than that without rebar coverage.

A comparison between the covered and uncovered trace void echoes at the same time is shown in Fig. 8. The amplitude is about $40 \mathrm{~V} / \mathrm{m}$ under no rebar coverage and decreases by $50 \%$ to $20 \mathrm{~V} / \mathrm{m}$ under rebar coverage.

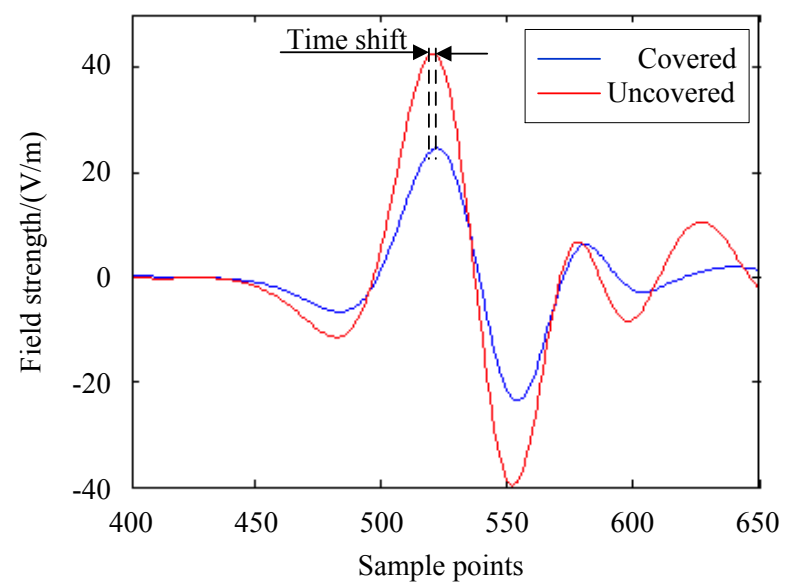

Fig. 8. Comparisons between the covered and uncovered void echo traces

Meanwhile, the signal echo at the lower part of rebar first arrives at the void disease surface through diffraction according to the Fermat theorem. The travel path is longer than that under no rebar coverage. In other words, a delay exists between the covered void echo and the uncovered void echo, as Fig. 8 shows.

2. Waveform overlapping

Fig. 5(a) illustrates that in the simulation calculation process, the antenna moves from the left to the right along the survey line. The direct distance between rebar and antenna differs with the location of antenna, a difference that is manifested by a hyperbolical characteristic curve on the GPR echo. Furthermore, considering that the ground background medium is a kind of loss medium, the amplitude of electromagnetic wave decreases with the increase of the propagation distance $(l)$ in the transmission process. An exponential relationship between $E$ and $l$ exists as well.

$|E|=E_{0} e^{-\alpha l / v}$

where $E$ is the electromagnetic wave amplitude, and when $l=0, E=E_{0}, v$ is the velocity of the electromagnetic wave, and $\alpha$ is the amplitude attenuation coefficient.

The rebar echo and void echo overlap when the time interval difference between them is smaller than the wavelet support interval. The comparisons between the covered and uncovered void echoes at the same space are shown in Fig. 9.

Red line in Fig. 9 is the void echo signal under no rebar coverage and the blue line is the void echo signal under rebar coverage. Fig. 9 shows that the echo amplitude in the rebar-covered region is smaller than that of the echo amplitude in the rebar-uncovered region, a finding that agrees with the above discussion results. Owing to the violent fluctuation at two sides of the coverage, the maximum amplitude in the rebar-covered region is larger than that in the rebar-uncovered region, which is attributed to the additive interference signal caused by the overlapping of rebar echo and void echo, which prevents the extraction of disease characteristics.

\subsection{Comparisons of different algorithms}

To compare the processing results of different algorithms, the frequency-domain deconvolution algorithm and the spiking deconvolution algorithm processed the simulation results in Section 3.2. Fig. 10 shows the processing results of different algorithms when $h_{1}=3 \mathrm{~cm}$ in Fig. 5(b).

A comparison between Figs. 10 and Fig. 6 suggests that the reflection coefficient returned by frequency-domain deconvolution algorithm has too many noise signals and cannot reflect interlayer positions. The spiking deconvolution algorithm fails to achieve the actual effect because the wavelet is in the non-minimum phase wavelet. According to Fig. 6, the subspace sparsity deconvolution algorithm can acquire accurate reflection layer information of the RC structure.

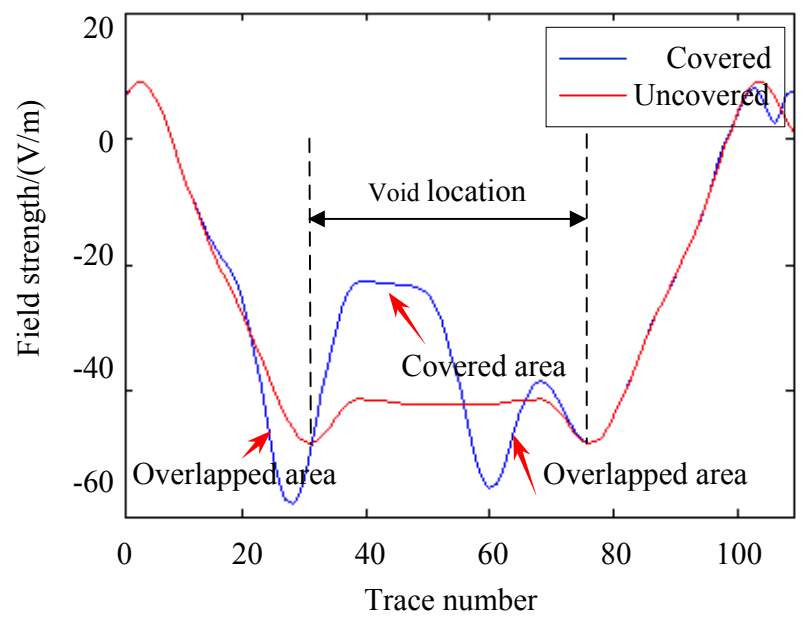

Fig. 9. Comparisons between the covered and uncovered void echo at the same space
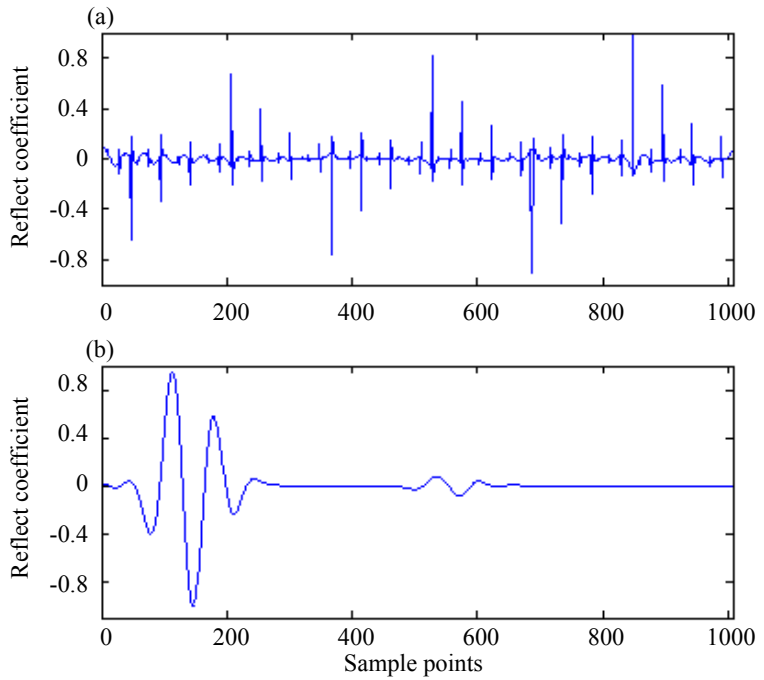

Fig. 10. Results with different deconvolution algorithms with trace number $=44$ and $h_{1}=3 \mathrm{~cm}$. (a) Processing result with frequency deconvolution; (b) Processing result with spiking deconvolution 


\subsection{Noise suppression}

To verify the noise suppression of the proposed algorithm, the white Gaussian noise (WGN) with different SNR (6-10 dB) was added to A-Scan echo signals in Fig. 5(b). The synthesis signals based on the original data when $S N R=10$ $\mathrm{dB}$ and $S N R=10 \mathrm{~dB}$ are shown in Fig. 11 .

In Fig. 11, the direct wave and rebar echo signal are distinct owing to the high intensity. However, the void echo is basically flooded by noise signal and its location cannot be identified accurately. The proposed algorithm processes echo data. When $S N R=10 \mathrm{~dB}$, the void echo processing result is equal to the the layer location without noise signal. When $S N R<10 \mathrm{~dB}$, the layer location shifts backward. When $S N R<6 \mathrm{~dB}$, the layer information is completely invalid. The processing results of void layer information under different SNR are shown in Table 2.

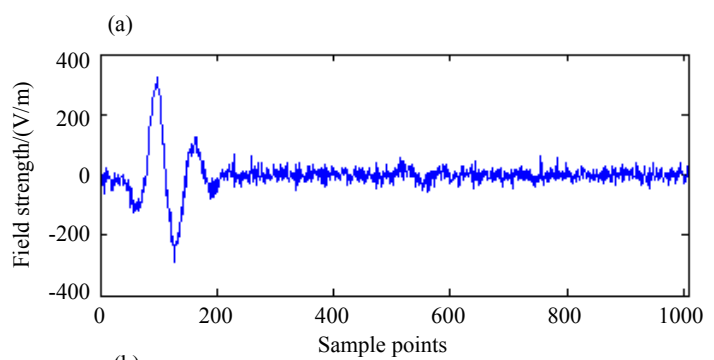

(b)

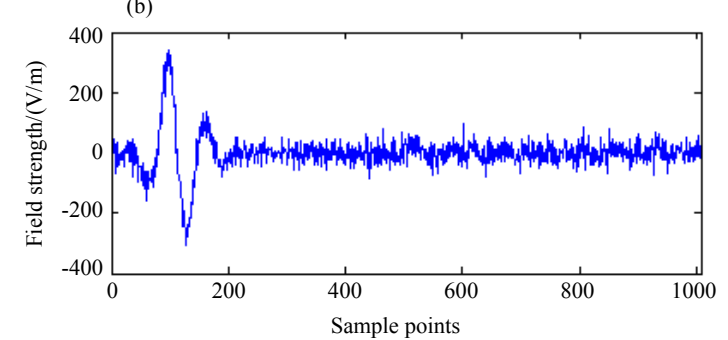

Fig. 11. Single trace data with $\mathrm{WGN}$ (a) $S N R=10 \mathrm{~dB}$; (b) $S N R=6 \mathrm{~dB}$

Table 2. Calculated void layer location with different WGN

\begin{tabular}{c|c|c}
\hline Index & SNR/(dB) & void location \\
\hline 1 & $\infty$ & 522 \\
2 & 10 & 522 \\
3 & 9 & 523 \\
4 & 8 & 525 \\
5 & 7 & 528 \\
6 & 6 & 528 \\
7 & 5 & $/$ \\
\hline
\end{tabular}

The applied WGN is the signal gained when the whole sampling interval has the same SNR. Given the high direct wave and rebar echo intensity, the energy intensity ratio between the applied WGN and the void echo is significantly higher than that of the whole sampling interval. According to the measured results, the SNR of WGN applied to the void echo is $-19.7 \mathrm{~dB}$ when the SNR of the whole sampling interval is $6 \mathrm{~dB}$, indicating that the proposed algorithm can acquire void layer location information accurately when local $S N R \geq-19.7 \mathrm{~dB}$.

\section{Conclusions}

The non-minimum phase deconvolution interlayer positioning and the echo enhancement algorithm were studied to enhance GPR echo signal in an RC medium. They were also conducted to overcome the contrast between the existing deconvolution algorithms' hypothesis of the minimum phase and the varying propagation characteristics of electromagnetic wave in loss medium. A multilayer medium model was established based on the equation of electromagnetic wave, and the wavelet attenuation characteristics of GPR electromagnetic wave under different conditions were described. The influences of rebar on lower void echo were analyzed and the subspace sparsity deconvolution algorithm was proposed. Next, the proposed algorithm was compared with other algorithms through experiments. Finally, the following conclusions are drawn:

(1) The influences of rebar on lower void echo signal are mainly manifested by the attenuation of disease echo intensity, echo delay, and echo overlapping. An exponential relationship between echo intensity and the two-way travel time exists. The longer the two-way travel time, the weaker the echo intensity. The void echo intensity is inversely proportional to the rebar diameter. Rebar diffraction brings a delay between covered and uncovered void echoes. The void echo and rebar echo overlap when the distance between rebar and the void disease is shorter than the wavelet, thus producing additive interference signal.

(2) The proposed subspace sparsity deconvolution algorithm does not depend on the minimum phase hypothesis and overcomes disagreement between existing algorithms (e.g., spiking deconvolution algorithm) and transmission characteristics of the electromagnetic wave of GPR. Moreover, it is applicable to acquire echo layer information under conditions with and without echo overlapping.

(3) The involvement of wavelet iteration update and time window subspace enhances interlayer echo signal and increases SNR quickly and effectively. The experiment demonstrates that the proposed algorithm can eliminate noise interference and acquire void layer location accurately when $S N R \geq-19.7 \mathrm{~dB}$.

The subspace sparsity deconvolution algorithm is proposed based on theoretical analysis and numerical simulation, which overcomes the restriction of the wavelet minimum phase of deconvolution algorithms. This algorithm can be used to enhance disease echo signal under rebar coverage in the multilayer RC structure, especially CA mortar void diseases in high-speed railway ballastless tracks. In actual processing, the echo signal of GPR is influenced by various factors. The high-speed railway ballastless track disease can be detected more accurately by combining the proposed algorithm and echo preprocessing.

\section{Acknowledgements}

This study was financially supported by the Railway Ministry of Science and Technology Research and Development Program of China (No. 2013G004-A-1) and Natural Science Foundation of Hebei Province under (No. E2017210113). 
Yang Yong, Du Yanliang, Zhao Weigang, Wang Baoxian and Zhao Hao/

Journal of Engineering Science and Technology Review 9 (6) (2016) 43-51

\section{References}

1. Ratto, C. R., Torrione, P. A., Collins L. M., "Exploiting GroundPenetrating Radar Phenomenology in a Context-Dependent Framework for Landmine Detection and Discrimination". IEEE Transactions on Geoscience \& Remote Sensing, 49(5), 2011, pp.1689 $-1700$.

2. Neal, A., "Ground-penetrating radar and its use in sedimentology: principles problems and progress". Earth-Science Reviews, 66(3), 2004, pp.261-330.

3. Donde, V., Pai, M. A., Hiskens I. A., "Simulation and optimization in an AGC system after deregulation. IEEE Power Engineering Review, 21(8), 2001, pp.58-58.

4. Fessant, T., "Gaussian-like tapered grating quarter wave-shifted DFB semiconductor lasers for high-power single-mode operation". Applied Physics B, 67(6),1998, pp.769-772.

5. Asare, V. D. S., "GPR detection of buried steel gas cylinder; a study conducted in a sandbox". International Journal of Basic \& Applied Sciences, 2(1), 2013, pp.64-72.

6. Bennia, A., Riad, S. M., "An optimization technique for iterative frequency-domain deconvolution". IEEE Transactions on Instrumentation \& Measurement, 39(2),1990, pp.358-362.

7. Sacchi, M. D., "Reweighting strategies in seismic deconvolution". Geophysical Journal International, 129(3), 1997, pp.651-656.

8. Canales, L. L., "Random noise reduction". Seg Technical Program Expanded Abstracts, 3(1), 1984, pp.329-329

9. Kim, J. H., Cho, S. J., Yi, M. J., "Removal of ringing noise in GPR data by signal processing". Geosciences Journal, 11(1), 2007, pp.7581.

10. Porsani, J. L., Sauck, W. A., "Ground-penetrating radar profiles over multiple steel tanks: Artifact removal through effective data processing”. Geophysics, 72(6), 2007, pp.77-83.

11. Pratt, W., Davarian, F., "Fast Computational Techniques for Pseudoinverse and Wiener Image Restoration". IEEE Transactions on Computers, 26(6), 1975, pp.571-580.
12. Wiggins, R. A., "Minimum entropy deconvolution". Geophysical Prospecting for Petrol, 16(1), 1980, pp.21-35.

13. Mcdonald, G. L., Zhao, Q., Zuo, M. J., "Maximum correlated Kurtosis deconvolution and application on gear tooth chip fault detection”. Mechanical Systems \& Signal Processing, 33(1), 2012, pp.237-255.

14 Donoho, D., “On Minimum entropy deconvolution”. Applied Time, 1981, pp.565-608.

15. Oppenheim, A. V., "Superposition in a class of nonlinear systems". IEEE Internat Convention Record part 1, 1964, pp.171-177.

16. Margrave, G. F., Lamoureux, M. P., "Gabor deconvolution". Cseg Annual Meeting, 21(1), 1999, pp.241-276.

17. Margrave, G. F., Lamoureux, M. P., Henley, D. C., "Gabor deconvolution: Estimating reflectivity by nonstationary deconvolution of seismic data". Geophysics, 76(3), 2011, pp.15-30.

18. Yee, K. S., Ingham, D., Shlager, K., "Time-domain extrapolation to the far field based on FDTD calculations". IEEE Transactions on Antennas \& Propagation, 39(3), 1991, pp.410-413.

19. Shangguan, P., Al-Qadi, I. L., "Calibration of FDTD Simulation of GPR Signal for Asphalt Pavement Compaction Monitoring”. IEEE Transactions on Geoscience \& Remote Sensing, 53(3), 2015, pp.1538-1548.

20. Bérenger, J. P., "On the Huygens absorbing boundary conditions for electromagnetics". Journal of Computational Physics, 226(1), 2007, pp.354-378.

21. Warren, C., Giannopoulos, A., Giannakis, I., “An advanced GPR modelling framework: The next generation of gprMax". International Workshop on Advanced Ground Penetrating Radar, Florence, Italy: IEEE, 2015, pp.1-4.

22. Giannakis, I., Giannopoulos, A., Warren, C., "A Realistic FDTD Numerical Modeling Framework of Ground Penetrating Radar for Landmine Detection". IEEE Journal of Selected Topics in Applied Earth Observations and Remote Sensing, 9(1), 2016, pp.37-51. 